\title{
Desenvolvimento de um MOOC Gamificado para Ensino de Bioinformática
}

\author{
Filipe B. S. das Neves ${ }^{1}$, Armando M. Toda ${ }^{4}$, André M. Ribeiro-dos-Santos ${ }^{2}$, \\ Ricardo Melo Casseb do Carmo ${ }^{1}$, Beatriz Stransky Ferreira ${ }^{3}$, Ândrea Ribeiro-dos- \\ Santos $^{2}$, Fabiano Cordeiro Moreira ${ }^{2}$ \\ ${ }^{1}$ Centro Universitário do Estado do Pará (CESUPA) - Unidade José Malcher - 66035- \\ 065 - Belém - PA - Brasil \\ ${ }^{2}$ Instituto de Ciências Biológicas - Universidade Federal do Pará (UFPA) - Laboratório \\ de Genética Humana e Médica Caixa Postal 8615 - 66075-970 - Belém - PA - Brasil \\ ${ }^{3}$ Universidade Federal do Rio Grande do Norte (UFRN) - Departamento de Engenharia \\ Biomédica - 59078970 - Natal, RN - Brasil \\ ${ }^{4}$ Instituto de Ciências Matemáticas e de Computação - Universidade de São Paulo \\ (USP) - Campus de São Carlos Caixa Postal 668 - 13560-970 - São Carlos - SP - \\ Brasil



Abstract. Bioinformatics is one of the multidisciplinary areas that emerged from technological advent, addressing concepts from Computation Sciences and Engineering resources to solve problems in Medical and Biological areas. These resources aid in the development of algorithms that perform expensive and complex processes, e.g., reading a sequencing experiment and the analysis in its bases. Professionals that work in Bioinformatics usually take on the main area of graduation, using the other as a background. By aiding in deconstruct this paradigm, this work aims at the development of a Gamified MOOC which aggregates contents related to Bioinformatics, using game elements to engage and motivate users.

Resumo. A Bioinformática é uma área multidisciplinar, fortemente impulsionada pelo avanço da tecnologia e geração de dados, que aplica conceitos e metodologias derivados da Ciência da Computação, Engenharia e Estatística para o estudo e solução de problemas nas áreas Médica e Biológicas. $O$ conhecimento proveniente das ciências exatas auxilia o desenvolvimento de ferramentas e algoritmos na realização de processos custosos e complexos, como a leitura de um experimento de sequenciamento, assim como a montagem e análise funcional de um genoma completo. Profissionais que atuam em Bioinformática geralmente assumem a área de formação, isto é, biomédica ou exatas, de modo que a outra torna-se segundo plano. Para auxiliar a aquisição de uma base mais sólida, que agrega conteúdos relacionados a área de Bioinformática, este trabalho visa demonstrar o desenvolvimento de um MOOC Gamificado, utilizando elementos de jogos para motivar e engajar os usuários. 
V Congresso Brasileiro de Informática na Educação (CBIE 2016)

Anais do XXVII Simpósio Brasileiro de Informática na Educação (SBIE 2016)

\section{Introdução}

Os avanços tecnológicos da última década permitiram o surgimento de diversas áreas, dentre elas a Bioinformática. Esta consiste no desenvolvimento e aplicação de ferramentas computacionais para gerenciar informações e solucionar problemas advindos da biologia, relacionados principalmente aos chamados "Omic data" (T.K. Attwood, 2011). Devido a esta natureza multidisciplinar, muitos profissionais que desejam iniciar nesta área podem apresentar dificuldades para assimilar os conteúdos, por serem discrepantes de sua área de formação (computação ou biociências).

Uma maneira de amenizar este problema é a utilização de Massive Online Open Courses (MOOCs), para que este aluno amplie seus conhecimentos e complemente sua formação na área. Os MOOCs são cursos ofertados em Ambientes Virtuais de Aprendizagem (AVAs) e podem alcançar um grande número de usuários. Por serem uma forma de educação à distância e muitas vezes auto-instrucionais, podem também apresentar a evasão massiva de seus usuários. Para contornar esta situação, algumas estratégias de motivação, como a gamificação, foram criadas. Esta estratégia é utilizada por exemplo na plataforma online Rosalind, que utiliza a gamificação para auxílio no ensino da Bioinformática (http://rosalind.info/about/). Esta concentra-se na resolução de problemas (Problem Based Learning), partindo do pressuposto que o indivíduo já tem um conhecimento prévio dos conceitos básicos.

Baseando-se nesta premissa, este trabalho visa apresentar o desenvolvimento de um MOOC com foco na Bioinformática. Este curso terá o diferencial de oferecer módulos introdutórios, com conteúdo pertinentes as duas áreas da interseção - biologia e computação, facilitando deste modo, a formação de novos profissionais que desejam ingressar na área.

\section{Referencial Teórico}

MOOCs surgiram em meados de 2008, no entanto só foram mundialmente difundidos em 2012, pelas inciativas tomadas por entidades como Coursera, Udacity e edX (Peco \& Luján-Mora, 2013). Estes sistemas, podem promover a consolidação de redes de aprendizagem sem limite de participantes (Zuquello \& Gimenes, 2015), e têm como principal objetivo aprimorar o aprendizado no ensino superior, podendo ser divididos em duas categorias: cMOOC e xMOOC (Balaji \& Sekhar, 2013).

Os cMOOCs se baseiam na abordagem conectivista, estabelecendo uma relação de "muitos-para-muitos" por meio da formação de grupos, fóruns de discussão, atividades colaborativas e relacionadas, além de não possuírem objetivos de aprendizagem específicos. Já os xMOOCs tratam da abordagem "um-para-muitos", onde o curso é conduzido por um instrutor e geralmente ocorre através do uso de uma plataforma (Balaji \& Sekhar, 2013).

Apesar desta diferença estrutural, ambos os cursos apresentam problemas relacionados a ausência de feedback em tempo real e a falta de motivação por parte dos estudantes (Hew \& Cheung, 2014), além da carência de documentos relacionados a sua estrutura interna (Zuquello \& Gimenes, 2015). Uma das maneiras de solucionar esta situação foi o desenvolvimento de estratégias de gamificação. Esta consiste em um processo de implementar elementos de jogos, fora de seu escopo natural, com o intuito 
V Congresso Brasileiro de Informática na Educação (CBIE 2016)

Anais do XXVII Simpósio Brasileiro de Informática na Educação (SBIE 2016)

de engajar, motivar e aprimorar experiências e o processo de aprendizagem do usuário final (Deterding, Sicart, Nacke, O'Hara, \& Dixon, 2011; Huotari \& Hamari, 2012; Kapp, 2012). O processo vem sendo amplamente utilizado no ensino, nos últimos anos, alcançando resultados positivos ( Borges, Durelli, Reis, \& Isotani, 2013).

\section{Desenvolvimento}

Para o desenvolvimento da plataforma, foi realizado um processo de coleta de requisitos funcionais em diversos MOOCs existentes como Coursera, edX, Udacity e Udemy, além da análise da plataforma Rosalind. Dentre os requisitos levantados, pode-se observar componentes para gerenciamento de cursos e materiais relacionados, usuários, dados e controle de qualidade, todos interligados por um módulo de interface.

Após a definição dos requisitos funcionais, foram gerados casos de uso para auxiliarem no desenvolvimento do sistema. Em seguida, foram estudadas as tecnologias (frameworks e linguagens de programação) para implementação do sistema. Baseado nos estudos realizados e na experiência da equipe, foi escolhida a linguagem Ruby, com o framework Ruby on Rails, devido a robustez e facilidade da ferramenta. A partir da documentação definida, foi gerada uma arquitetura inicial com os módulos do sistema (Figura 1). Estes módulos são interligados, compartilhando informações entre eles.

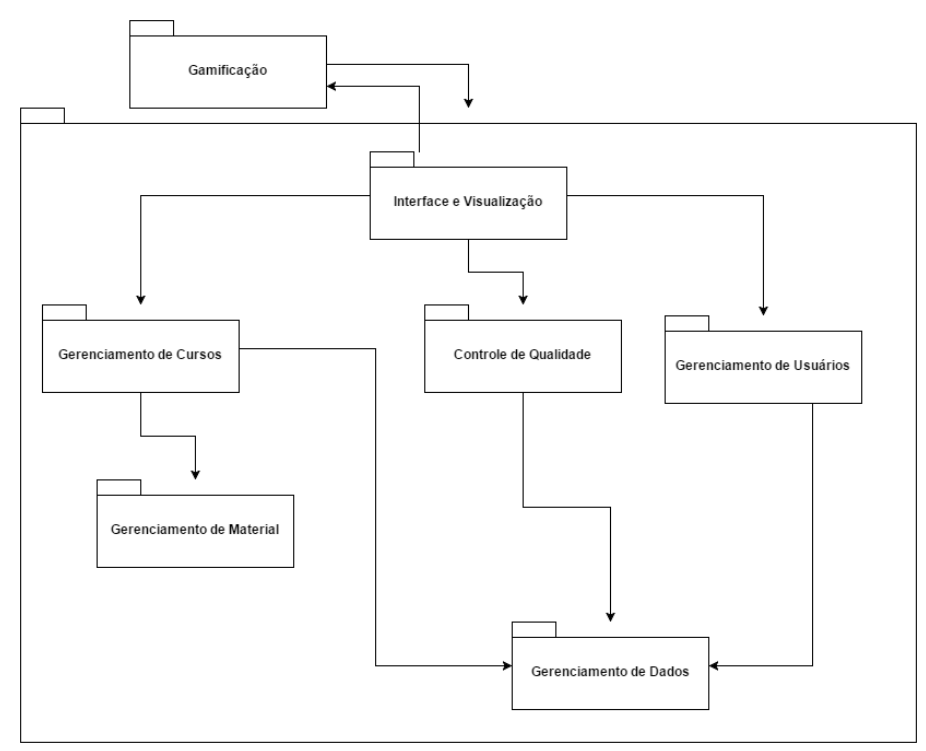

Figura 1. Representação de alto nível do sistema, em diagrama de pacotes.

Foi escolhida uma representação de alto nível, assim como um sistema modular, devido ao fato de poder acrescentar novos módulos, um destes módulos são os elementos de gamificação, que serão incluídos quando o protótipo estiver concluído e funcional.

A gamificação do sistema será utilizada como um módulo externo, recebendo informações do módulo de Interface e Visualização e alimentando o sistema com os elementos de jogos. Exemplos da interação do módulo de gamificação com os demais poderá ser visualizada no Controle de Qualidade, onde os usuários realizarão um feedback do curso inscrito e das atividades contidas, este retorno será visualizado pelo docente responsável (que aumenta ou diminui a sua reputação) e impactado no perfil do aluno (que recebe benefícios por realizar esta ação). 
V Congresso Brasileiro de Informática na Educação (CBIE 2016)

Anais do XXVII Simpósio Brasileiro de Informática na Educação (SBIE 2016)

\section{Resultado Preliminares}

Até o momento, foram obtidos os documentos de software com requisitos e diagramas UML, e um modelo da arquitetura de referência do sistema, incluindo algumas interações dos elementos de gamificação com os demais módulos. A Figura 2 apresenta um caso de uso, de alto nível, indicando 1 usuário com funções padrão (Usuário Padrão) e suas duas especialidades, podendo ser um Aluno ou um Administrador do Sistema (que, inicialmente, será responsável pela manutenção e inserção dos cursos).

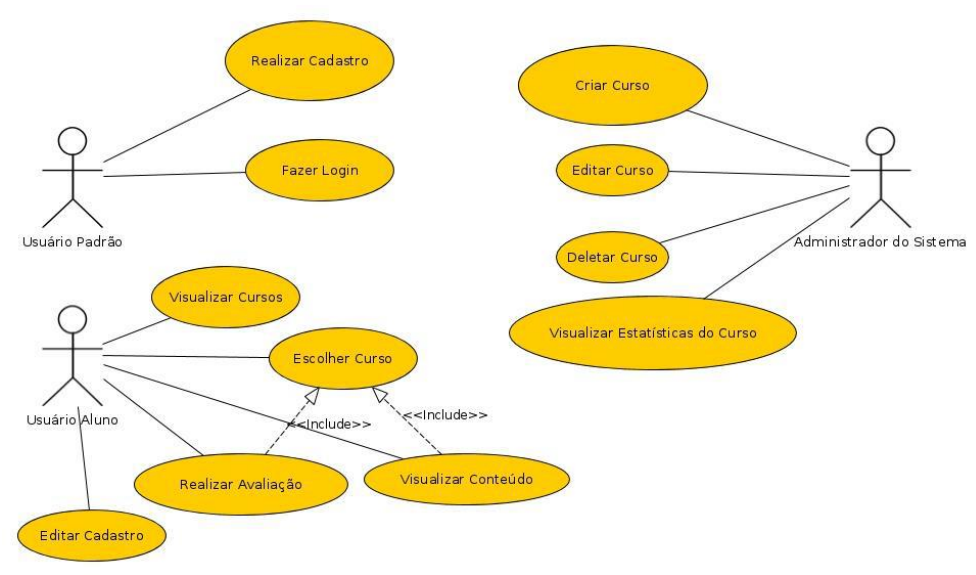

Figura 2. Caso de uso de alto nível das funcionalidades do sistema

Dentre as interações gamificadas, pode-se citar o sistema de pontos (mecanismo para ser utilizado junto com o sistema de feedback das atividades) e o quadro de conquistas (elementos adquiridos pelos usuários que desempenharem ações específicas dentro do sistema).

Além disso, foram gerados formulários de cadastro para usuários e professores, assim como a estrutura inicial de cursos, onde um docente pode criar um curso e atribuir atividades objetivas. Em paralelo, a equipe de docentes interdisciplinar está gerando os conteúdos que serão abordados (das áreas básicas de Computação e Biologia), de forma que sigam uma estratégia pedagógica aceitável.

Por fim, o banco de dados está sendo gerado de modo que possa armazenar uma grande quantidade de dados, sem que influencie no desempenho do sistema (considerando um grande número de usuários). Além da escalabilidade, os autores também geraram a base considerando os dados estatísticos a serem coletados, como o tempo que o usuário utiliza para finalizar uma atividade, tópico ou curso. Estes dados têm como objetivo auxiliar na preparação e edição dos conteúdos pedagógicos no sistema.

\section{Conclusões e Trabalhos Futuros}

Este trabalho apresentou o desenvolvimento inicial de um MOOC gamificado, focado na área de Bioinformática. Acredita-se que este trabalho é importante por explorar a área de MOOCs, e por não ter sido encontrado, até o momento, um sistema gamificado desse porte focado na área de Bioinformática, considerando que a Rosalind é uma plataforma focada na resolução de problemas. 
V Congresso Brasileiro de Informática na Educação (CBIE 2016)

Anais do XXVII Simpósio Brasileiro de Informática na Educação (SBIE 2016)

Como trabalhos futuros, além do término da implementação, pretende-se avaliar se os elementos de gamificação podem auxiliar e influenciar significativamente no aprendizado inicial dos profissionais de Bioinformática. Pretende-se também proceder com a análise individual dos elementos de gamificação, de modo a otimizar o efeito no processo de aprendizagem.

\section{Agradecimentos}

This study was supported by CAPES (Coordenacão de Aperfeicoamento Pessoal de Nível Superior) - Rede de Pesquisa em Genomica Populacional Humana (RPGPH) 051/2013-CAPES-BioComputacional. The funders had no role in the study design, data collection and analysis, decision to publish, or preparation of the manuscript, and FAPESP (processo 2016/02765-2).

\section{Referências}

Balaji, B. S., \& Sekhar, A. C. (2013). The various facets of MOOC. In 2013 IEEE International Conference in MOOC, Innovation and Technology in Education (MITE) (pp. 139-142). IEEE. http://doi.org/10.1109/MITE.2013.6756322

Borges, S. S., Durelli, V. H. S., Reis, H. M., \& Isotani, S. (2013). Gamificação Aplicada à Educação : Um Mapeamento Sistemático. Anais Do Simpósio Brasileiro de Informática Educativa, (July 2015), 234-243. http://doi.org/10.5753/CBIE.SBIE.2013.234.

Deterding, S., Sicart, M., Nacke, L., O’Hara, K., \& Dixon, D. (2011). Gamification. using game-design elements in non-gaming contexts. Proceedings of the 2011 Annual Conference Extended Abstracts on Human Factors in Computing Systems CHI EA '11, 2425. http://doi.org/10.1145/1979742.1979575.

Hew, K. F., \& Cheung, W. S. (2014). Students' and instructors' use of massive open online courses (MOOCs): Motivations and challenges. Educational Research Review, 12, 45-58. http://doi.org/10.1016/j.edurev.2014.05.001Smith, A. and Jones, B. (1999). On the complexity of computing. In Advances in Computer Science, pages 555-566. Publishing Press.

Huotari, K., \& Hamari, J. (2012). Defining gamification: a service marketing perspective. Proceeding of the 16th International Academic MindTrek Conference, 17-22. Retrieved from http://dl.acm.org/citation.cfm?id=2393137

Kapp, K. M. (2012). The Gamification of Learning and Instruction: Game-based Methods and Strategies for Training and Education. Retrieved from http://dl.acm.org/citation.cfm?id=2378737

Peco, P., \& Luján-Mora, S. (2013). Architecture of a MOOC based on CourseBuilder. ... Based Higher Education and ... Retrieved from http://ieeexplore.ieee.org/xpls/abs_all.jsp?arnumber=6671045

T.K. Attwood, A. G. N.-E. E. and E. B.-R. (2011). Bioinformatics - Trends and Methodologies. (M. A. Mahdavi, Ed.). InTech. http://doi.org/10.5772/786

Zuquello, A. G., \& Gimenes, I. M. de S. (2015). OERecommender : um Sistema de Recomendação de REA para MOOC, (Laclo), 331-340. 